\title{
Developmental Changes in the Organization of Functional Connections between the Basal Ganglia and Cerebral Cortex
}

\author{
Deanna J. Greene, ${ }^{1,2}$ Timothy 0. Laumann, ${ }^{3}$ Joseph W. Dubis, ${ }^{3}$ S. Katie Ihnen, ${ }^{3}$ Maital Neta, ${ }^{3}$ Jonathan D. Power, ${ }^{3}$ \\ John R. Pruett Jr., ${ }^{1}$ Kevin J. Black, ${ }^{1,2,3,4}$ and Bradley L. Schlaggar ${ }^{2,3,4,5}$ \\ Departments of ${ }^{1}$ Psychiatry, ${ }^{2}$ Radiology, ${ }^{3}$ Neurology, ${ }^{4}$ Anatomy \& Neurobiology, and ${ }^{5}$ Pediatrics, Washington University School of Medicine, St. Louis, \\ Missouri 63110
}

The basal ganglia (BG) comprise a set of subcortical nuclei with sensorimotor, cognitive, and limbic subdivisions, indicative of functional organization. BG dysfunction in several developmental disorders suggests the importance of the healthy maturation of these structures. However, few studies have investigated the development of BG functional organization. Using resting-state functional connectivity MRI (rs-fcMRI), we compared human child and adult functional connectivity of the BG with rs-fcMRI-defined cortical systems. Because children move more than adults, customized preprocessing, including volume censoring, was used to minimize motion-induced rsfcMRI artifact. Our results demonstrated functional organization in the adult BG consistent with subdivisions previously identified in anatomical tracing studies. Group comparisons revealed a developmental shift in bilateral posterior putamen/pallidum clusters from preferential connectivity with the somatomotor "face" system in childhood to preferential connectivity with control/attention systems (frontoparietal, ventral attention) in adulthood. This shift was due to a decline in the functional connectivity of these clusters with the somatomotor face system over development, and no change with control/attention systems. Applying multivariate pattern analysis, we were able to reliably classify individuals as children or adults based on BG-cortical system functional connectivity. Interrogation of the features driving this classification revealed, in addition to the somatomotor face system, contributions by the orbitofrontal, auditory, and somatomotor hand systems. These results demonstrate that BG-cortical functional connectivity evolves over development, and may lend insight into developmental disorders that involve BG dysfunction, particularly those involving motor systems (e.g., Tourette syndrome).

Key words: basal ganglia; development; functional connectivity; resting state

\section{Introduction}

The basal ganglia (BG) comprise a set of subcortical nuclei that are involved in motor control, and executive and limbic functions (Middleton and Strick, 2000; McClure et al., 2003; Gerardin et al., 2004; Aron et al., 2007; Graybiel, 2008). Dysfunction in BG structures and their cortical connections has been implicated in movement disorders, including Tourette syndrome (TS) and Parkinson disease, and in disorders of executive control, including attention-deficit/hyperactivity disorder (ADHD) and obsessive-

Received July 19, 2013; revised March 10, 2014; accepted March 18, 2014.

Author contributions:D.J.G., K.J.B., and B.L.S. designed research;D.J.G., J.W.D., S.K.I., M.N., and J.R.P. performed research; J.D.P. contributed unpublished reagents/analytic tools; D.J.G., T.O.L., and K.J.B. analyzed data; D.J.G. wrote the paper.

This project was supported by National Institutes of Health (NIH) Grants R21MH091512 (B.L.S.), R01HD057076 (B.L.S.), and K24MH087913 (K.J.B.); a Tourette Syndrome Association fellowship (D.J.G.), the Intellectual and Developmental Disabilities Research Center at Washington University (NIH/National Institute of Child Health and Human Development Grant P30 HD062171); NIH Grant K12 EY016336 (J.R.P.); and The Simons Foundation Autism Research Initiative (for "Brain Circuitry in Simplex Autism" study, to Steven E. Petersen, investigator). We thank Steven E. Petersen, Deanna Barch, and Judith Lieu for the contribution of data; Jonathan Koller for assistance with figures; Babatunde Adeyemo for assistance with figures and support vector machine scripts; and Abraham Z. Snyder for consultation on methods.

The authors declare no competing financial interests.

Correspondence should be addressed to Deanna J. Greene, East Building, Neuroimaging Laboratories, $4525 \mathrm{Scott}$ Avenue, Suite 2220, St. Louis, M0 63110. E-mail: greened@npg.wustl.edu.

DOI:10.1523/JNEUROSCI.3069-13.2014

Copyright $\odot 2014$ the authors $\quad 0270-6474 / 14 / 345842-13 \$ 15.00 / 0$ compulsive disorder (OCD; Bradshaw and Sheppard, 2000). Interestingly, several of these disorders (e.g., TS, ADHD, OCD) develop during childhood. Thus, understanding the development of the BG and their functional cortical connections in humans would be beneficial not only to developmental cognitive neuroscience, but also to child neurology and psychiatry.

Most knowledge of BG connectivity is based on anatomical tracing and single-unit studies in nonhuman primates (Alexander et al., 1986; Middleton and Strick, 2000). The data converge on spatially specific corticostriatal connections: somatosensorimotor regions project primarily to the posterior putamen (in a somatotopically organized fashion); prefrontal and other "association" regions project primarily to the caudate and anterior putamen; and limbic/paralimbic regions project primarily to the ventral striatum (Selemon and Goldman-Rakic, 1985; Alexander et al., 1986; Parent, 1990; Haber, 2003; Nambu, 2011). Neuroimaging studies, implementing diffusion tensor imaging and resting state-functional connectivity MRI (rs-fcMRI), have revealed that the organization of anatomical and functional connectivity between the BG and the cortex in healthy adult humans corresponds well with nonhuman primate data (Lehéricy et al., 2004; Leh et al., 2007; Di Martino et al., 2008; Draganski et al., 2008; Barnes et al., 2010; Choi et al., 2012). However, there is surprisingly little research on the development of corticostriatal 
functional connectivity. Given the role of the BG in several developmental disorders, it is of particular interest to investigate BG connectivity during the developmental period in which these disorders typically begin or peak in severity. For example, the onset of TS and ADHD is typically around 6-7 years of age. Studying the development of functional connectivity patterns from this early age may be key for understanding the mechanisms that go awry in such developmental disorders.

Here, we interrogated the development of functional connectivity between the BG and cerebral cortex using rs-fcMRI. Our goal was to generate a context within which we could pursue investigations of atypically developing populations with presumed dysfunction in cortical-BG interactions. In particular, we wished to identify developmental changes in BG functional organization by examining, in healthy children and adults, patterns of blood oxygenation level-dependent (BOLD) correlations between the BG and cortical networks previously defined by rsfcMRI (hereinafter referred to as "systems"). We hypothesized that the maturation of BG functional organization would reflect a developmental shift toward greater involvement of control systems in the brain (Casey et al., 2005). First, we applied traditional univariate analyses to capture group differences. Second, we applied multivariate pattern analysis (MVPA) tools to classify individuals as children or adults based on patterns of BG functional connectivity and to detect features of BG development that may not have been captured by the univariate analyses.

\section{Materials and Methods \\ Participants}

A group of 120 healthy young adults (19-31 years old; 60 males) and 60 healthy children (7-12 years old; 33 males) were selected from an extant database of participants ( $n=487$; age, 6.7-35.4 years old; 206 males) on the basis of having at least $5.5 \mathrm{~min}$ of rs-fcMRI data after motion "scrubbing" (see below). Participants had been recruited from the Washington University campus and the surrounding community. All participants were native English speakers and right handed. Participants reported no history of neurological or psychiatric disease and were not taking psychotropic medications, as assessed by self-report for adult participants and parental report for child participants. All adult participants, and a parent or guardian for each child participant, gave informed consent, and all children assented. All participants were compensated for their participation. The Washington University Human Research Protection Office approved all studies.

\section{Image acquisition}

Data were acquired on a Siemens 3 T MAGNETOM Trio scanner with a Siemens 12-channel Head Matrix Coil. To help stabilize head position, each subject was fitted with a thermoplastic mask fastened to holders on the head coil. A T1-weighted sagittal MP-RAGE structural image (slice time echo, $3.06 \mathrm{~ms}$; TR, $2.4 \mathrm{~s}$; inversion time, $1 \mathrm{~s}$; flip angle, $8^{\circ}$; 127 slices; $1 \times 1 \times 1 \mathrm{~mm}$ voxels) and a T2-weighted turbo spin echo structural image (TE, $84 \mathrm{~ms}$; TR, $6.8 \mathrm{~s}$; 32 slices; $2 \times 1 \times 4 \mathrm{~mm}$ voxels) in the same anatomical plane as the BOLD images were obtained to improve alignment to an atlas. Functional images were acquired using a BOLD contrast-sensitive echo planar sequence (TE, $27 \mathrm{~ms}$; flip angle, $90^{\circ}$; inplane resolution, $4 \times 4 \mathrm{~mm}$; volume TR, $2.5 \mathrm{~s}$ for 177 participants; TR, $2.2 \mathrm{~s}$ for 3 child participants). Whole-brain coverage was obtained with 32 contiguous interleaved $4 \mathrm{~mm}$ axial slices. Steady-state magnetization was assumed after four volumes. In the adult group, $336 \pm 121$ functional volumes (range, 184-724) were acquired, and in the child group, $377 \pm$ 166 functional volumes (range, 260-780) were acquired.

During functional "resting" scans, participants viewed a centrally presented white crosshair (subtending a $<1^{\circ}$ visual angle) on a black background. Participants were instructed to relax, watch the crosshair, and hold as still as possible.

\section{Image analysis}

Preprocessing. Functional images from each subject were preprocessed to reduce artifacts (Shulman et al., 2010). These steps included the following: (1) sinc interpolation of all slices to the temporal midpoint of the first slice, accounting for differences in the acquisition time of each individual slice; (2) correction for head movement within and across runs; and (3) intensity normalization to a whole-brain mode value (across voxels and TRs) of 1000 for each run. Atlas transformation of the functional data was computed for each individual via the MP-RAGE scan. Each run was then resampled in atlas space on an isotropic $3 \mathrm{~mm}$ grid combining movement correction and atlas transformation in a single interpolation (Shulman et al., 2010). The target atlas (TRIO_KY_NDC, http://nrg.wikispaces.com/atlas_targets) was created using validated methods (Black et al., 2004) from MP-RAGE scans from thirteen 7- to 9-year-old children (seven males) and twelve 21- to 30-year-old adults (six males). The scans for the atlas were collected on the same Siemens 3 T MAGNETOM Trio scanner used in the present study. The atlas was made to conform to the Talairach atlas space as defined by the spatial normalization method of Lancaster et al. (1995). The image registration methods used here, even when applied to images with 8-10 $\mathrm{mm}$ resolution (FWHM), are accurate to within $\leq 1 \mathrm{~mm}$ (Black et al., 2001; Rowland et al., 2005).

Functional connectivity preprocessing. For rs-fcMRI data, several additional preprocessing steps were applied to reduce spurious variance (e.g., heart rate and respiration) unlikely to reflect neuronal activity (Fox et al., 2009). These steps included a multiple regression of nuisance variables from the BOLD data and a temporal bandpass filter $(0.009 \mathrm{~Hz}<\mathrm{f}<0.08$ $\mathrm{Hz})$. Nuisance regressors included individualized ventricular and white matter signals averaged from regions of interest (ROIs) defined from each participant's structural image, six detrended head realignment parameters obtained by rigid-body head motion correction, and the derivatives of these signals and parameters. Global signal was not included as a nuisance regressor, as the partial correlation approach implemented in our analyses (see below) accounted for the shared variance among cortical systems.

Motion scrubbing. Head motion can cause spurious yet systematic changes in BOLD correlations, affecting group comparisons (Power et al., 2012; Van Dijk et al., 2012). Thus, the data in this report underwent a volume censoring (scrubbing) procedure similar to that previously described (Power et al., 2012, 2014) to minimize motion-related effects. Frame-by-frame head displacement (FD) was calculated from preprocessing realignment estimates, and frame-by-frame signal change [root mean square value of all brain voxels at every volume of the differentiated BOLD time series (DVARS)] was calculated from the functional connectivity image generated after functional connectivity preprocessing. A temporal mask was formed by flagging any volume with DVARS $>2 \%$ BOLD signal change, and any volume with FD $>0.25 \mathrm{~mm}$, as well as two volumes before and two volumes after the FD-flagged volumes to account for spin history effects and possible temporal spread of artifactual signal during temporal filtering. The data were then reprocessed using the temporal masks that excluded all flagged volumes so that motioncontaminated data contributed neither to regressions nor to functional connectivity estimates. This procedure removed $18 \pm 14 \%$ of the data from the 120 adults, leaving $272 \pm 105$ volumes (range, 150-719) of usable data per subject, and it removed $30 \pm 17 \%$ of the data from the 60 children, leaving $257 \pm 111$ volumes (range, 132-622) of data per subject. Though the number of volumes removed differed in the children and the adults, the number of remaining (analyzed) volumes did not $(p=0.37)$, and differences in the amount of censoring have not appreciably affected group comparisons of adult rs-fcMRI data (discussed in Power et al., 2014). The partial correlation approach in the present analyses (which has a similar effect on motion artifact to that of global signal regression) accounts for the temporally remote effects of a movementtainted frame on functional connectivity (e.g., the prolonged effects lasting many seconds after motion, as noted in Power et al., 2014).

\section{Anatomical definitions}

Basal ganglia definition. Segmentation of basal ganglia structures was performed using FSL version 4.1.8 [Oxford Centre for Functional Mag- 
netic Resonance Imaging of the Brain (FMRIB) Software Library (www.fmrib.ox.ac.uk/fsl)]. MP-RAGE structural images were entered into the FMRIB integrated registration and segmentation tool (FIRST), which defines eight subcortical regions: caudate nucleus, putamen, pallidum, nucleus accumbens, amygdala, hippocampus, thalamus, and brainstem. The caudate, putamen, pallidum, and nucleus accumbens were grouped together and isolated to create a basal ganglia mask for each subject. Those voxels labeled as basal ganglia in $75 \%$ of our total subject pool were included in the group basal ganglia mask, as this overlap provided reasonable borders of basal ganglia structures of interest in the child and adult cohorts (Fig. 1A).

Cortical system definition. To investigate functional connectivity between the basal ganglia and known cortical functional systems, we selected cortical systems from a brain network scheme that was created by applying graph theoretical and community detection tools to rsfcMRI data (Power et al., 2011). For this scheme, a full voxelwise approach for graph definition (in which each voxel in the brain is treated as a node in the graph) was modified such that short-distance connections were excluded. This "modified voxelwise" approach used by Power et al., 2011 generated a map of putative brain network modules that corresponds well with known functional systems in the brain (i.e., brain systems previously identified by task-activation studies). From this whole-brain description of functional network organization, we selected major systems with reliable representation and particularly well defined functional significance. These cortical systems encompassed most cortical regions with known/suspected anatomical connections to the basal ganglia (Alexander et al., 1986; Haber and Knutson, 2010). These systems, however, did not include the orbitofrontal cortex, which has known connections to the ventral striatum. Therefore, we also included an orbitofrontal system derived from analyses in Power et al. (2011). This process resulted in a set of 11 systems (Fig. 1B): (1) sensory and somatomotor systems: visual, auditory, somatomotor "face," and somatomotor "hand";

(2) attention/control systems: frontoparietal, cingulo-opercular, dorsal attention, ventral attention, and salience; (3) the default mode system; and (4) the orbitofrontal system.

\section{Correlations between cortical systems and the basal ganglia}

Partial correlation analyses. For each subject, the mean BOLD time series was extracted from each of the 11 cortical systems. The partial correlation was then calculated, as in Zhang et al. (2008), between the BOLD time series in each basal ganglia voxel and each cortical system (i.e., for each system and BG voxel, a residual correlation was computed after partialling out the signal from the other cortical systems). These partial correlations were then normalized using Fisher's $r$-to- $z$ transform, and averaged across a group of subjects (e.g., 120 adults, 60 children). Only positive correlations were used for subsequent group and winner-take-all analyses. To test the significance of the partial correlations, we computed $t$ statistics for each voxel and converted the $t$ statistics to $z$-scores.

Regressing out cortical signal adjacent to the basal ganglia. Anatomically, some basal ganglia structures are immediately adjacent to cortical regions within the 11 selected cortical systems. For example, the putamen is just medial to the insula, which contributes to the cingulo-opercular, sa- lience, and auditory systems. It is possible that signal bleed between adjacent regions could lead to artifactual increases in correlation strength between voxels in lateral putamen and the cortical systems that include insula. To account for this possibility, we identified those voxels belonging to the 11 cortical systems of interest that were spatially located within $5 \mathrm{~mm}$ of the basal ganglia mask (i.e., the set of voxels that overlapped between the cortical systems mask and a $5 \mathrm{~mm}$ dilated version of our basal ganglia mask). This procedure identified 128 voxels, most of which were in the insula, with some others in medial prefrontal cortex. Similar to Choi et al. (2012), we regressed out the mean signal of those specific cortical voxels from the BOLD data within the basal ganglia, to specifically avoid possible shared signal from nearby cortical regions. All main results include this regression. Where specifically indicated, control analyses are performed without this regression.

Group comparisons. For purposes of comparing adults and children, we divided the group of 120 adults into two cohorts of 60 , each of which was compared with the 60 children. The two adult cohorts and the child group were matched on the number of volumes retained after motionscrubbing procedures (see Table 1 for subject characteristics). We directly compared the partial correlation coefficients of children and adults within each cortical system by computing two-sample $t$ tests (accounting for unequal group variance) on the Fisher $z$-transformed $r$ values for each cortical system. We then corrected for multiple comparisons using false discovery rate (FDR; $p<0.05)$ and a minimum cluster size of 10 voxels. To explore the functional connectivity profile of voxel clusters surviving correction for multiple comparisons, we created seeds ( $5 \mathrm{~mm}$ radius) centered on the clusters to generate seed maps in each group. Wholebrain correlation maps for each subject were generated, and then each 
Table 1. Participant demographic information

\begin{tabular}{llll}
\hline & $\begin{array}{l}\text { Age } \\
\text { (years) }\end{array}$ & $\begin{array}{l}\text { Male/ } \\
\text { female }(n)\end{array}$ & $\begin{array}{l}\text { Volumes retained after } \\
\text { motion scrubbing }\end{array}$ \\
\hline Adult cohort 1 $(n=60)$ & $24.8(2.4)$ & $30 / 30$ & $272.4(95.8)$ \\
Adult cohort 2 $(n=60)$ & $24.7(2.4)$ & $30 / 30$ & $272.2(113.8)$ \\
Children $(n=60)$ & $10.5(1.3)$ & $33 / 27$ & $256.8(110.8)$ \\
\hline
\end{tabular}

Data are mean (SD), unless otherwise indicated. There were no significant differences between groups on sex ( $p=$ 0.58 ) or volumes retained ( $p=0.41 ; p=0.45$ ).

subject's $z$-transformed correlation map was used to generate grouplevel whole-brain correlation maps for each adult cohort and for the child group. The correlation maps of the child group were compared to each adult cohort to produce images describing any differences.

Winner-take-all analyses. We applied a winner-take-all approach to parcellate the basal ganglia according to their functional relationships with cortical systems. This approach has previously provided reasonable functional parcellations of several subcortical brain structures (Zhang et al., 2008; Fair et al., 2010; Buckner et al., 2011; Choi et al., 2012), and provides an image for summarizing the relative strengths of functional relationships between a structure and multiple brain systems. In the present analyses, each basal ganglia voxel was assigned to the cortical system with which it correlated most (displayed using corresponding colors). For display purposes, all images have been resampled to $1 \times 1 \times$ $1 \mathrm{~mm}$ space within the basal ganglia mask. To compare winner-take-all maps across groups, we calculated the number of voxels that differed by winner assignment. To quantify statistical significance, we used permutation testing on 120 subjects (60 adults and 60 children), randomly selecting 60 subjects and comparing them to the remaining 60 subjects in each permutation (100,000 permutations). This procedure estimated the distribution of the number of voxels differing by winner assignment between two equal groups of 60 adults and children chosen by chance. Then, the true number of voxels differing by winner assignment between the 60 children and each of the two previously defined adult cohorts of 60 was computed and compared against the distribution from the permutation testing to assess significance.

\section{Classification using multivariate pattern analysis}

We used MVPA, specifically support vector machine (SVM) algorithms, to test how well the functional relationships between the BG and cortical systems could be leveraged to classify an individual's maturation status (i.e., child or adult). An SVM takes a training set of labeled samples, each with a series of features, and learns the properties and weights of the features that characterize the labels. Once a decision function is learned based on the training data, it can be used to predict the class label of a new, previously unseen, test sample (Schölkopf and Smola, 2002; BenHur et al., 2008).

We used the Spider Machine Learning Toolbox in Matlab (http://www. kyb.mpg.de/bs/people/spider/) for our SVM computations and applied methods previously used in our laboratory (Dosenbach et al., 2010). Briefly, each subject was treated as a point in multidimensional space where the number of dimensions equals the number of features. Entering the partial correlation matrix (781 voxels $\times 11$ cortical systems $\times 120$ subjects) into the SVM, we treated each functional connection ( $r$ value) between a given BG voxel and a given cortical system as a feature, resulting in 8591 features. Using soft-margin separation (Ben-Hur et al., 2008), linear kernel SVMs were applied to distinguish between subjects belonging to two classes: child and adult. Separate SVMs were run to distinguish children from each of the previously defined adult cohorts. To estimate classification accuracy and to identify the most consistent discriminating features, we used a leave-one-out-cross-validation (LOOCV) method, where each subject was designated as the test sample in turn, while the remaining samples were used to train the SVM classifier. As each subject was designated as the test sample once, there were as many folds (rounds of cross-validation) as subjects. Within each LOOCV fold, feature reduction was applied, as this technique has been shown to speed up computation and improve classification performance (Hastie et al., 2001; De Martino et al., 2008; Pereira et al., 2009). The number of features was reduced to 120 , equal to our number of samples/subjects, since decre- ments in performance arise when the number of features substantially exceeds the number of training samples due to overfitting. As in Dosenbach et al. (2010), two-sample $t$ tests (not assuming equal variance) were run to compare the features across the two classes on the training set of each LOOCV fold (with the test sample excluded). The features with the highest $t$ scores were retained. Once all folds were completed, the accuracies for all folds were averaged together to generate a final accuracy estimate (percentage of subjects accurately classified), and the one-tailed probability of the accuracy estimate (under the null hypothesis that each subject assignment was random with a probability of 0.5 ) was calculated using the binomial distribution. To interrogate the features that most contributed to the classification, those features that were retained in all LOOCV folds ( $100 \%$ consensus) for each SVM were extracted. We were specifically interested in consensus features that were similar across both SVM classifiers (i.e., child vs adult cohort 1 , and child vs adult cohort 2).

\section{Results}

\section{Changes in basal ganglia functional connectivity over development}

Partial correlations in adults

Adult partial correlation maps within the basal ganglia for each of the 11 cortical systems are shown in Figure 2, and peak coordinates are listed in Table 2. While some of the partial correlation results were consistent with known anatomical connections, rsfcMRI correlations do not necessitate monosynaptic connections (Vincent et al., 2007). Functional connectivity can provide unique information about the functional relationships between brain regions, as demonstrated by several results here.

The somatomotor hand system correlated with the dorsal posterior putamen, while the somatomotor face system correlated with the ventral posterior putamen, showing a dorsal-to-ventral topography consistent with known anatomical connections from tracing studies in nonhuman primates (Alexander and DeLong, 1985; Flaherty and Graybiel, 1993; Nambu, 2011). The auditory system also showed correlations with the ventral posterior putamen, yet its highest correlations were within the head of the caudate. The visual system correlated with the ventral striatum and anterior putamen, as well as with posterior portions of the pallidum. The orbitofrontal system strongly correlated with the ventral striatum, consistent with tracing studies in nonhuman primates (Haber and McFarland, 1999; Haber, 2003).

Examination of the spatial patterns of partial correlation can provide insight into the extent to which there is overlapping functional organization across cortical systems. For instance, the sensory and motor systems just described demonstrated spatially segregated connectivity in the BG, whereas the attention/control systems and the default-mode system demonstrated some spatial overlap in their BG connectivity. The frontoparietal and ventral attention systems largely overlapped, correlating with the caudate (extending from the head to the body) and with large portions of the putamen extending into the pallidum. The default and dorsal attention systems also correlated with the caudate, but extended into the ventral striatum. These systems showed some segregation, as the default system additionally correlated with the ventral pallidum, while the dorsal attention system correlated with the ventral anterior putamen. The salience system overlapped largely with the frontoparietal and ventral attention systems along the body and head of the caudate, as well as with the default and dorsal attention systems in the caudate and ventral striatum. The salience system also correlated with the anterior parts of the pallidum. Finally, the cingulo-opercular system correlated with large portions of the putamen and pallidum. 


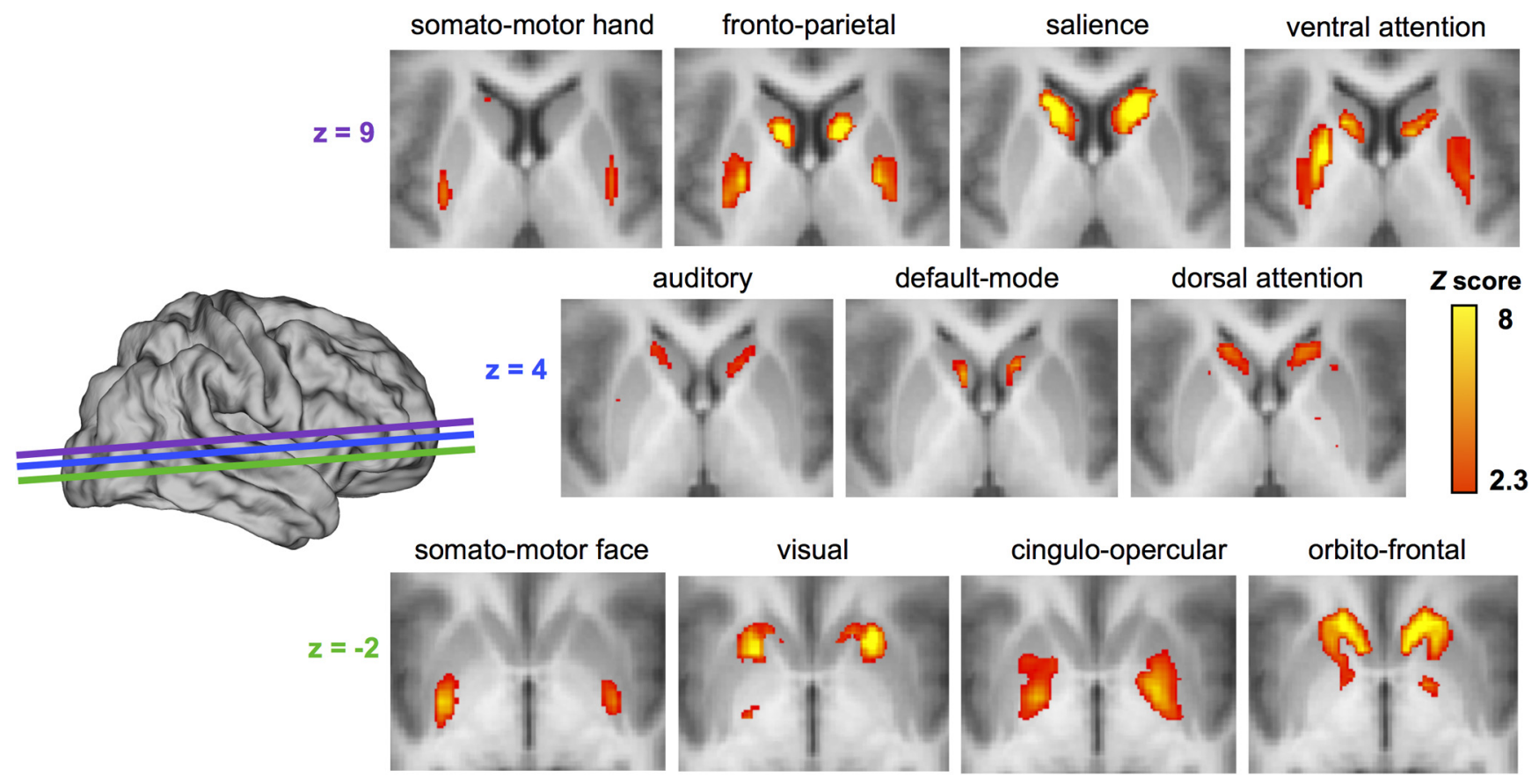

Figure 2. Partial correlation maps for each cortical system in the adult group $(n=120)$. Purple, blue, and green lines displayed across the cortex on the left correspond to the transverse slices (in the z-plane) shown here. Slices were chosen that best represent the peak partial correlation values between basal ganglia voxels and each cortical system. Significant partial correlations are defined as those with a $z$-score $>2.3(p<0.01)$. Image left is anatomical left.

\section{Group comparisons of partial correlations}

The partial correlation results in the children were largely similar to those in adults, demonstrating an overall highly comparable spatial organization. However, the children appeared to have larger-magnitude correlations with the somatomotor face system (Fig. 3A). Direct statistical comparisons of the partial correlations confirmed that age group differences were found selectively with the somatomotor face system, and were localized to a cluster of voxels in the posterior putamen and pallidum (Fig. 3B). These voxels showed significantly greater correlations with the somatomotor face system in children than in adults. Direct statistical comparison did not reveal a significant age group difference in the correlation strengths between any basal ganglia voxels and the other cortical systems.

Within the posterior putamen/pallidum cluster that showed a significant group difference, we further examined whether there was a relationship between the partial correlations and age. We calculated the Pearson correlation between age and the mean partial correlation coefficient of all the voxels in the cluster for the somatomotor face system, separately for the children and adults. Figure $3 C$ shows that there was a significant correlation in the children $(r=-0.31, p=0.014)$, as the partial correlation decreased with age, but there was no significant correlation in the adults $(r=0.09, p=0.33)$. Thus, the correlation with the somatomotor face system decreased in childhood and had stabilized by early adulthood. As we suspect that a great deal of dynamics occurs developmentally within the child group age range (7-12 years), a more detailed analysis of development within this range would be interesting but is underpowered in the current study.

Seed maps of the regions showing group differences

To explore the bilateral posterior putamen/pallidum regions that differed between children and adults, we examined the functional connectivity profiles of those regions. Seed maps for the left hemisphere cluster (cluster center MNI coordinates: $-26,-11$, $-1)$ for each group are shown in Figure 4A. Seed maps for the right hemisphere cluster (cluster center MNI coordinates: 29, $-11,-2)$ look very similar but have higher magnitudes. Group comparisons showed that the correlation maps differed between children and adults in somatomotor cortex bilaterally, specifically within the somatomotor face system (Fig. $4 B$; results were the same for the right hemisphere seed). Correlations between the seed regions and the somatomotor face area were greater in children than in adults, as expected by the partial correlation results.

\section{Winner-take-all results}

To create winner-take-all maps, each basal ganglia voxel was labeled by the cortical system with which it correlated the most (Fig. 5), as in Zhang et al. (2008). In the adults, the auditory (pink), dorsal attention (bright green), and default mode (red) systems were not well represented in the winner-take-all maps, as they correlated more strongly than the other cortical systems in only a few basal ganglia voxels. Of the systems that were well represented, there was a posterior-to-anterior topography in the caudate nucleus from ventral attention (teal) to salience (black), which then moved into the orbitofrontal system (cream) in the ventral striatum. There were also ventral portions along the caudate represented by the frontoparietal (yellow) system. In the posterior putamen, the ventral-dorsal topography of the somatomotor face (orange) and hand (light blue) systems was clearly demonstrated. In addition to these somatomotor systems, control systems were represented in the putamen, including the frontoparietal (yellow), ventral attention (teal), and cinguloopercular (purple) systems. Finally, in the pallidum, control systems and the visual system (dark blue) were represented most strongly. Thus, consistent with a previous report (Choi et al., 2012), we have observed functional organization within the basal ganglia in typical adults using rs-fcMRI-derived functional sys- 
Table 2. Positive partial correlations of the basal ganglia with each cortical system

\begin{tabular}{|c|c|c|c|c|c|c|}
\hline \multirow[b]{2}{*}{ Cortical system } & \multirow[b]{2}{*}{ Anatomical region } & \multirow[b]{2}{*}{ Side } & \multicolumn{3}{|c|}{ MNI coordinates } & \multirow{2}{*}{$\begin{array}{l}\text { Peak } \\
Z \text {-score }\end{array}$} \\
\hline & & & $x$ & $y$ & $z$ & \\
\hline \multirow[t]{3}{*}{ Auditory } & Caudate (anterior) & $\mathrm{L}$ & -11 & 19 & 5 & 4.88 \\
\hline & & $\mathrm{R}$ & 9 & 13 & 5 & 4.26 \\
\hline & Putamen (posterior, ventral) & $\mathrm{R}$ & 29 & -5 & -7 & 4.26 \\
\hline \multirow[t]{2}{*}{ Somatomotor face } & Putamen (posterior, ventral) & L & -29 & -9 & -4 & 6.92 \\
\hline & & $\mathrm{R}$ & 29 & -8 & -5 & 4.95 \\
\hline \multirow[t]{2}{*}{ Somatomotor hand } & Putamen (posterior, dorsal) & L & -29 & -11 & 7 & 4.51 \\
\hline & & $\mathrm{R}$ & 30 & -8 & 8 & 5.11 \\
\hline \multirow[t]{6}{*}{ Visual } & Pallidum & L & -21 & -10 & 2 & 4.90 \\
\hline & & $\mathrm{R}$ & 25 & -10 & 2 & 4.72 \\
\hline & Putamen (anterior, ventral) & $\mathrm{L}$ & -20 & 11 & -5 & 5.95 \\
\hline & & $\mathrm{R}$ & 20 & 14 & -6 & 6.97 \\
\hline & Ventral striatum & $\mathrm{L}$ & -10 & 16 & 0 & 5.69 \\
\hline & & $\mathrm{R}$ & 11 & 17 & -2 & 6.03 \\
\hline \multirow[t]{4}{*}{ Cingulo-opercular } & Pallidum & L & -20 & -6 & -4 & 6.65 \\
\hline & & $\mathrm{R}$ & 20 & -4 & -5 & 6.87 \\
\hline & Putamen & $\mathrm{L}$ & -24 & 9 & 1 & 6.38 \\
\hline & & $\mathrm{R}$ & 24 & 9 & 7 & 6.60 \\
\hline \multirow[t]{5}{*}{ Dorsal attention } & Caudate & $\mathrm{R}$ & 17 & 18 & 10 & 3.96 \\
\hline & Putamen (anterior, ventral) & $\mathrm{L}$ & -20 & 17 & -8 & 4.24 \\
\hline & & $\mathrm{R}$ & 20 & 13 & -9 & 4.49 \\
\hline & Ventral striatum & $\mathrm{L}$ & -14 & 21 & 0 & 5.46 \\
\hline & & $\mathrm{R}$ & 12 & 21 & 2 & 5.61 \\
\hline \multirow[t]{4}{*}{ Frontoparietal } & Caudate & L & -10 & 11 & 8 & 7.87 \\
\hline & & $\mathrm{R}$ & 12 & 12 & 9 & 8.13 \\
\hline & Putamen & L & -23 & -4 & 5 & 5.46 \\
\hline & & $\mathrm{R}$ & 26 & -5 & 3 & 5.51 \\
\hline \multirow[t]{6}{*}{ Salience } & Caudate & L & -13 & 17 & 4 & 10.58 \\
\hline & & $\mathrm{R}$ & 15 & 18 & 5 & 11.35 \\
\hline & Pallidum (anterior) & L & -17 & 3 & 2 & 5.17 \\
\hline & & $\mathrm{R}$ & 15 & 2 & -1 & 4.89 \\
\hline & Ventral striatum & L & -18 & 12 & -4 & 6.44 \\
\hline & & $\mathrm{R}$ & 11 & 14 & -2 & 7.33 \\
\hline \multirow[t]{5}{*}{ Ventral attention } & Caudate & L & -13 & 10 & 13 & 10.88 \\
\hline & & $\mathrm{R}$ & 15 & 7 & 16 & 9.34 \\
\hline & Putamen & L & -27 & -8 & 1 & 6.58 \\
\hline & & L & -21 & 2 & 6 & 9.13 \\
\hline & & $\mathrm{R}$ & 23 & 7 & 2 & 5.64 \\
\hline \multirow[t]{6}{*}{ Default mode } & Caudate & L & -11 & 9 & 15 & 4.73 \\
\hline & & $\mathrm{R}$ & 12 & 9 & 15 & 4.64 \\
\hline & Pallidum (ventral) & L & -19 & -6 & -5 & 3.42 \\
\hline & & $\mathrm{R}$ & 22 & -4 & -6 & 3.6 \\
\hline & Ventral striatum & L & -9 & 13 & -2 & 5.55 \\
\hline & & $\mathrm{R}$ & 10 & 17 & 0 & 4.61 \\
\hline \multirow[t]{2}{*}{ Orbitofrontal } & Ventral striatum & L & -15 & 18 & -6 & 8.23 \\
\hline & & $\mathrm{R}$ & 19 & 15 & -9 & 9.23 \\
\hline
\end{tabular}

$L$, left; $R$, right.

tems. Further, the winner-take-all maps were similar across different cohorts of adults (i.e., when we divided the 120 adults into two groups of 60; data not shown). These winner-take-all maps demonstrate that this functional parcellation approach reveals organization in the basal ganglia that is not evident in the structural MRI MP-RAGE images alone.

Visual examination of the winner-take-all maps in children suggests similarities to the adults in the caudate and ventral striatum, and some differences from the adults in the putamen (Fig. $5)$. The general organization of the control systems in the caudate and the dominance of the orbitofrontal system in the ventral striatum were preserved in children and adults. However, the somatomotor systems and the auditory system "won" in the putamen in children, while control systems, specifically the ventral attention and frontoparietal systems, won in the putamen in adults. The total number of voxels that differed by winner assignment between child and adult groups was significantly higher than would be expected by chance. As shown in Figure 3D, the child group differed from one adult cohort in 388 voxels (of 781 possible) and from the other adult cohort in 368 voxels (both comparisons $p<0.001$ by permutation testing).

Justification for regression of cortical signals near the basal ganglia When winner-take-all analyses were conducted without regressing out the signal from cortical voxels anatomically adjacent to the basal ganglia, marked changes from the results above were seen: almost all of the putamen correlated most strongly with the cingulo-opercular system. The cingulo-opercular system includes the anterior insula, which directly neighbors the putamen. Thus, the high correlations between voxels in the putamen and the cingulo-opercular system in this analysis approach may be due to signal bleed between the putamen and insula. This result supports the decision to remove such signal for the primary analyses, though such an approach reduces our ability to detect true correlations that might exist between the basal ganglia and nearby cortex (specifically, cortex in the cingulo-opercular system).

\section{Reliability of winner assignments}

To assess the reliability of winner assignments in the winnertake-all maps, we conducted the winner-take-all analysis for each individual subject and then calculated the number of subjects in agreement upon the winner for each basal ganglia voxel. In the adults, there was high reliability in the dorsal caudate and ventral striatum, and low reliability in the putamen and pallidum (Fig. $6 A$, adults). In other words, reliability was highest for assigning the control systems to the caudate and the orbitofrontal system to the ventral striatum. In addition, we examined the margin by which the winning system correlated with each basal ganglia voxel more than the system in second place (i.e., $\Delta r$ ) on the group level. This group-level analysis yielded highly similar results to the subject-level reliability analysis (Fig. $6 B$, adults), as the dorsal caudate and ventral striatum had the largest $\Delta r$ values.

In the children, reliability was highest in the caudate and ventral striatum (Fig. 6A, children), similar to the adults. However, unlike the adults, reliability was also high in the posterior putamen/pallidum bilaterally in the children. These regions spatially corresponded to the regions that showed significant differences between children and adults. Thus, the winning relationship in the posterior putamen/pallidum with the somatomotor face system in the children was highly reliable, but the relationship in the same region with control systems in the adults was less reliable. The group-level $\Delta r$ maps (showing the difference between the correlation with the winning cortical system and the system in second place) demonstrated the same pattern as the reliability maps (Fig. 6B, children).

\section{Investigating possible analysis confounds}

The significant difference between children and adults replicated when we conducted the analysis without regressing out the cortical signal adjacent to the basal ganglia, demonstrating that this regression did not influence the group difference results. We also investigated a subset of 45 children and 45 adults who were matched on the precise number of males and females (23 males per group), number of volumes retained for analysis (children: mean $=249.4, \mathrm{SD}=79.8$; adults: mean $=250.4, \mathrm{SD}=78.3 ; p=$ 0.95 ), and movement (FD) after motion scrubbing (children: mean $=0.11, \mathrm{SD}=0.016$; adults: mean $=0.11, \mathrm{SD}=0.01 ; p=$ $0.34)$. Group comparisons of these highly matched subgroups replicated our results, demonstrating significantly greater correlations in the posterior putamen/pallidum with the somatomotor face sys- 
A

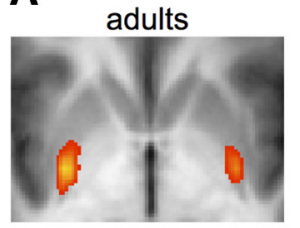

children

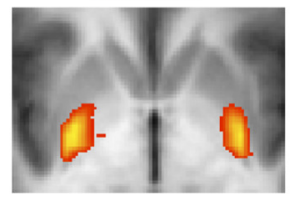

$z=-2$

C

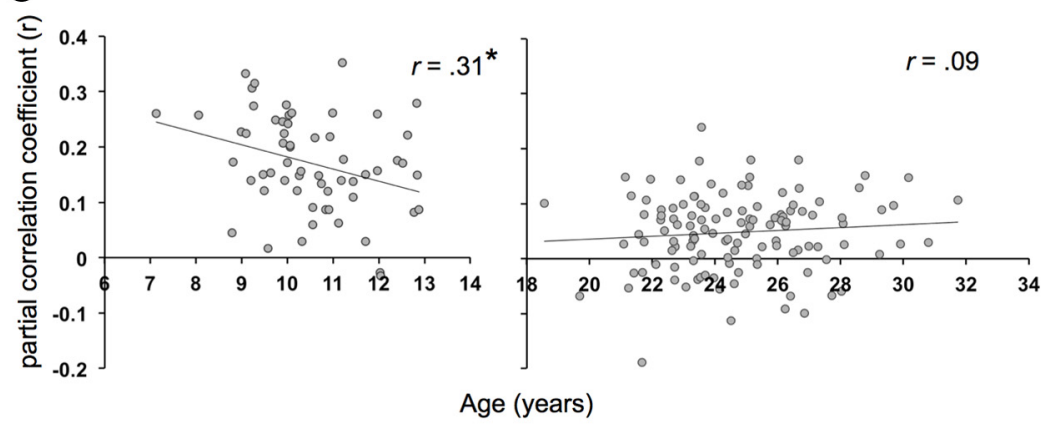

children vs. adult cohort 1
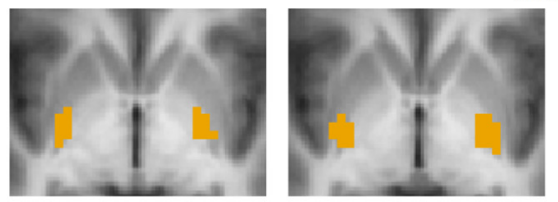
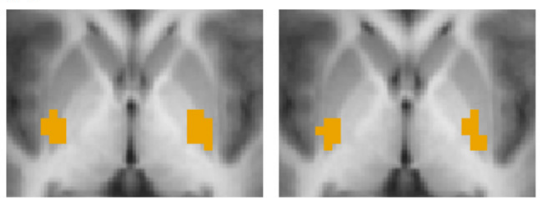

children vs. adult cohort 2

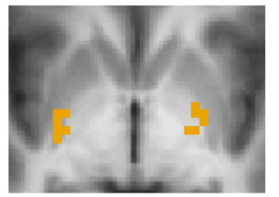

$z=-2$

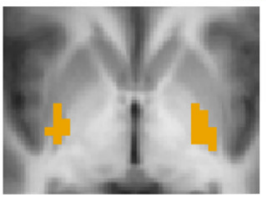

$z=-1$

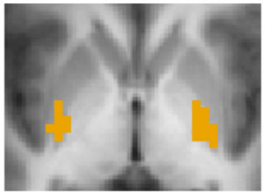

$z=0$

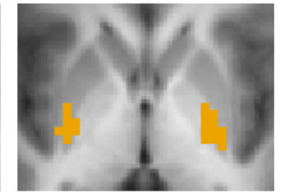

$z=1$

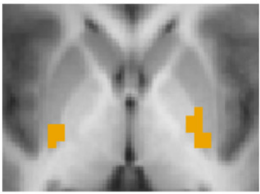

$z=2$

D

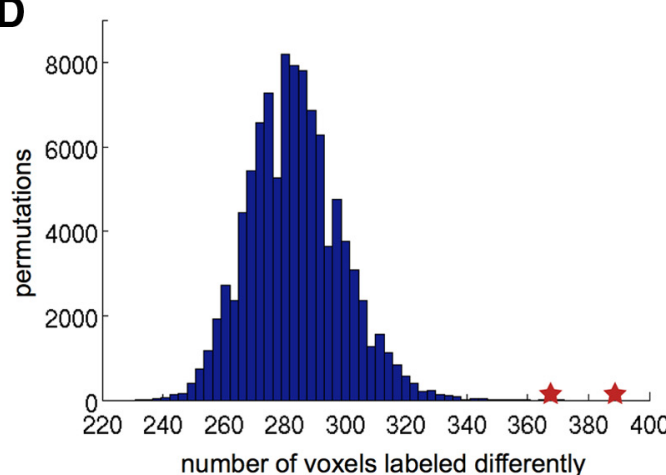

Figure 3. Children differ significantly from adults. A, Partial correlations for the somatomotor face system in adults and children; $Z$-score scale is 2.3-8, as in Figure 2. B, Voxels with a significant difference in partial correlations between children and each adult cohort; colored orange to correspond with the somatomotor face system. $C$, Correlation between age and mean partial correlation coefficient within the voxels colored in $\boldsymbol{B}$. ${ }^{*}$ Significance. $\boldsymbol{D}$, Frequency histogram showing the number of voxels labeled differently (in winner-take-all assignment) across 100,000 random assignments of data in two groups composed of children and adults (blue). The number of voxels labeled differently between the 60 children and the two original adults cohorts shown by the red stars.

tem in children than in adults, confirming that these results were not driven by residual group differences. Direct comparisons between the two adult cohorts revealed no significant differences in correlation strength with any cortical system, as expected.

To further investigate whether residual differences in motion (or the neural sources of motion) may have affected functional correlations, we compared seed maps of five somatomotor face ROIs (from Power et al., 2011) between the child and adult cohorts. If differences in the neural sources of face and mouth movements were present and affected correlations between the basal ganglia and cortex, we would expect that correlations between homotopic somatomotor regions would also differ between children and adults. Whole-brain seed-map comparisons showed no significant group differences in cortical voxels (twosample $t$ tests, FDR corrected, $p<0.05$, but not cluster size corrected to be more liberal). When we narrowed the space to compare seed maps specifically within the somatomotor face system, there were no significant differences, with the exception of two voxels in one of the seed-map comparisons in the child versus adult cohort 1 comparison, but not in the child versus adult cohort 2 comparison (seed MNI coordinates: -39, -11, 35). Thus, we did not find compelling evidence for differences in homotopic functional correlations.

We also tested whether our result might be explained by anatomical differences (i.e., whether the voxels identified by the group comparison were mostly putamen in one group and mostly pallidum in the other). Such an anatomical explanation was not supported. Of the 76 voxels showing group differences in the child cohort versus adult cohort 1 comparison, 25 voxels were labeled by the segmentation tool as putamen and 49 as pallidum in the child cohort (2 voxels were labeled as something else), and 27 were labeled as putamen and 47 as pallidum in the adult cohort. These anatomical identifications were not significantly different: $\chi^{2}(2, N=120)=0.63(p=0.731)$. Of the 66 voxels showing group differences in the child cohort versus adult cohort 2 comparison, 20 were labeled as putamen and 45 as pallidum in the child cohort, and 22 were labeled as putamen and 43 as pallidum in the adult cohort, which again was not significantly different: $\chi^{2}(2, N=120)=0.69(p=0.708)$.

Signal dropout was assessed in the basal ganglia in the child and adult groups by examining the mean BOLD signal, calculated as the average of the first frame of acquisition (after steady-state magnetization) from each group of subjects (Ojemann et al., 1997; Wig et al., 2013). Within the basal ganglia mask, voxels with $>20 \%$ signal loss (using the same conservative threshold as in Wig et al., 2013) were found in the inferior part of the ventral striatum, consistent with known susceptibility artifacts in the nucleus accumbens; the adults had more signal loss in these voxels than the children. Thus, caution is warranted when interpreting results in the inferior part of the ventral striatum (which correlated with the orbitofrontal system). It is worth noting, however, that the partial correlations and winner-take-all assignments did not differ between children and adults in these ventral striatum voxels. Voxels elsewhere in the basal ganglia did not demonstrate signal loss $>20 \%$ in either group. 


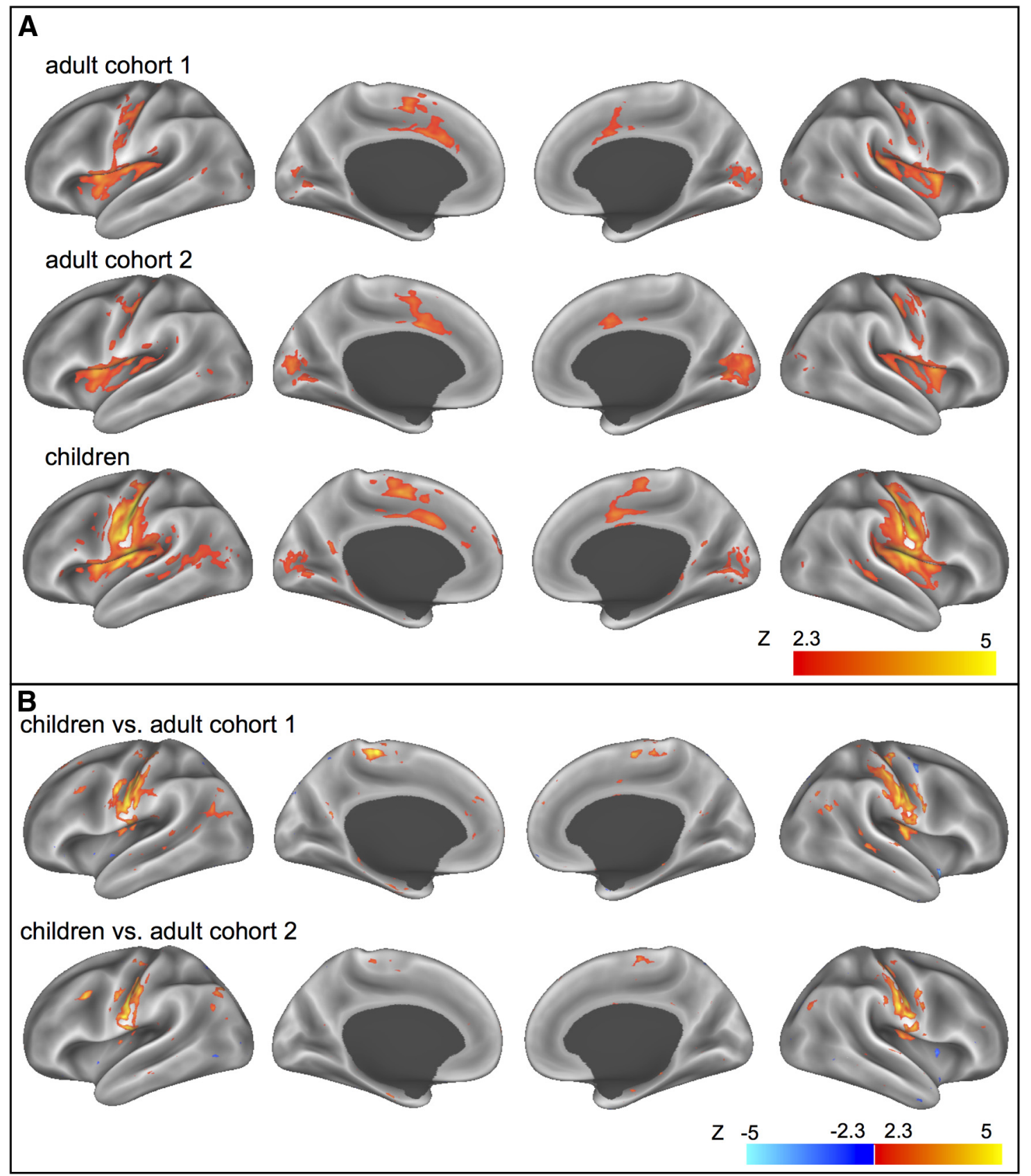

Figure 4. A, Whole-brain functional connectivity of left hemisphere putamen/pallidum seed (MNI coordinates: $-26,-11,-1)$ in children $(n=60)$ and each adult cohort ( $n=60$ each). Positive correlations are displayed in warm colors. $\boldsymbol{B}$, Comparisons of each adult cohort to the cohort of children. Stronger correlations in children than adults are displayed in warm colors; stronger correlations in adults than children are displayed in cool colors.

SVM reliably classifies maturation status based on basal ganglia-cortical system functional connectivity

Classification accuracy

SVM classification of individual subjects as children or adults based on basal ganglia-cortical system functional connectivity was $83.3 \%$ accurate (sensitivity, $81.7 \%$; specificity, $85 \%$; $p=$ $2.8 \times 10^{-14}$ ) for classifying children and adult cohort 1 , and $75 \%$ accurate (sensitivity, $73.3 \%$; specificity, $76.7 \% ; p=1.9 \times 10^{-8}$ ) for classifying children and adult cohort 2 . To check that SVM classifiers were not able to make distinctions between groups that should not be different, we also divided the 60 children and 60 adults in cohort 1 into two groups of 60 subjects, with 30 children and 30 adults per group, according to whether their subject number was odd or even. Subject numbers were randomly assigned on the day of the MRI scanning session, and therefore should not reflect a systematic difference related to physiology. SVM classification of odd or even subject numbers was $53.3 \%$ accurate, which was not significant $(p=0.262)$. The SVM was also not able to reliably classify subjects from the two adult cohorts labeled either by cohort ( $45 \%$ accuracy, $p=0.882)$ or by sex $(55.8 \%$ accuracy, $p=0.118$ ). Thus, the classifiers were able to detect patterns in the functional relationships between the basal ganglia and cortical systems that distinguished children and adults, but not other groupings.

Important features driving the developmental distinctions

We extracted the features that were among the most heavily weighted across every LOOCV fold (i.e., 100\% consensus), and 
Adults
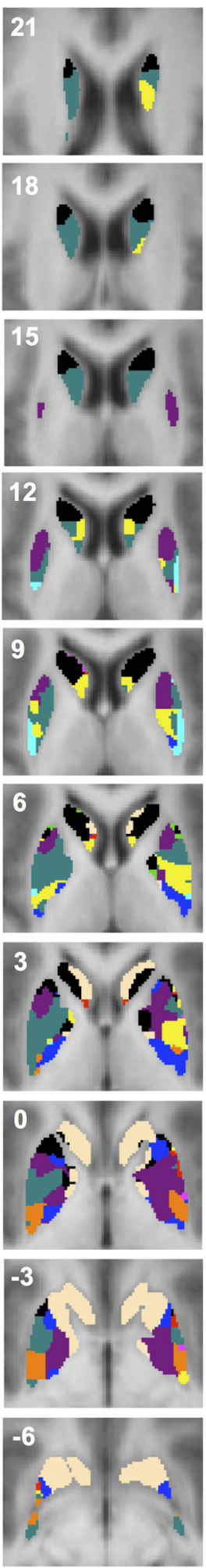

Children
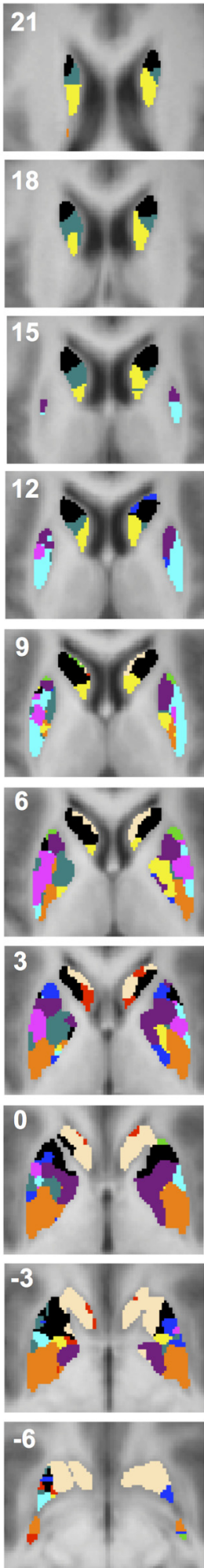

Adults
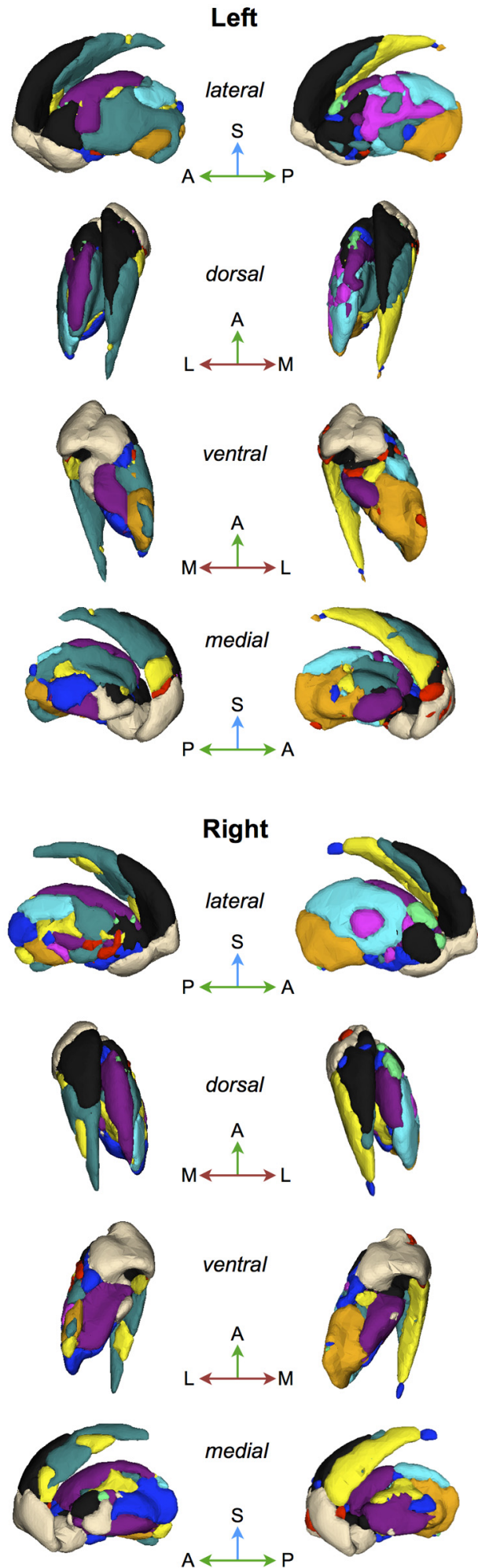

$\square$ auditory $\square$ somato-motor hand $\square$ fronto-parietal $\square$ dorsal attention $\square$ salience $\square$ orbitofrontal
$\square$ visual $\quad \square$ somato-motor face $\square$ cingulo-opercular $\square$ ventral attention $\square$ default-mode

Figure 5. Winner-take-all maps in adults and children, displaying functional connectivity between the basal ganglia and functionally defined cortical systems. Each basal ganglia voxel is colored according to the cortical system it most correlates with (color key at the bottom). On the left, slices shown in the transverse plane (image left is anatomical left), $z=-7$ to $z=22$ (top left corner). On the right, 3-D renderings displayed in lateral, dorsal, ventral, and medial views. Superior (S) direction is shown with a blue arrow; anterior (A) and posterior (P) directions are shown with a green arrow; and medial $(\mathrm{M})$ and lateral $(\mathrm{L})$ directions are shown with a red arrow. 
A adults
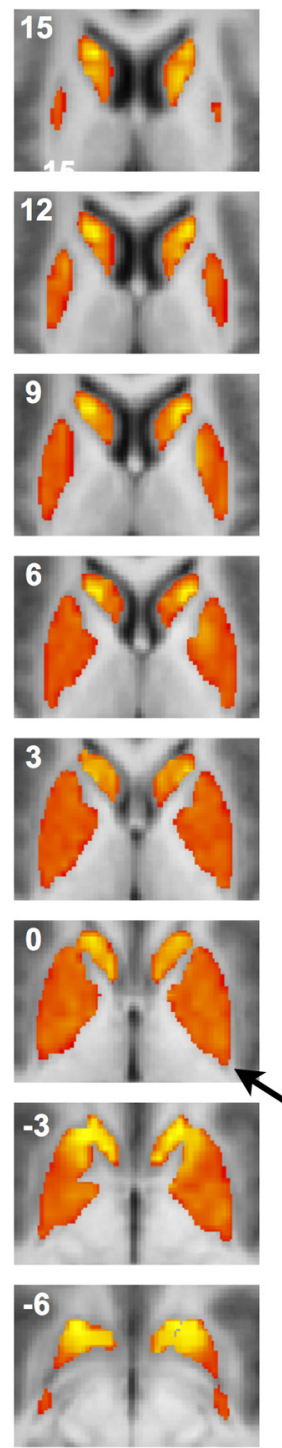

$0 \%$ children
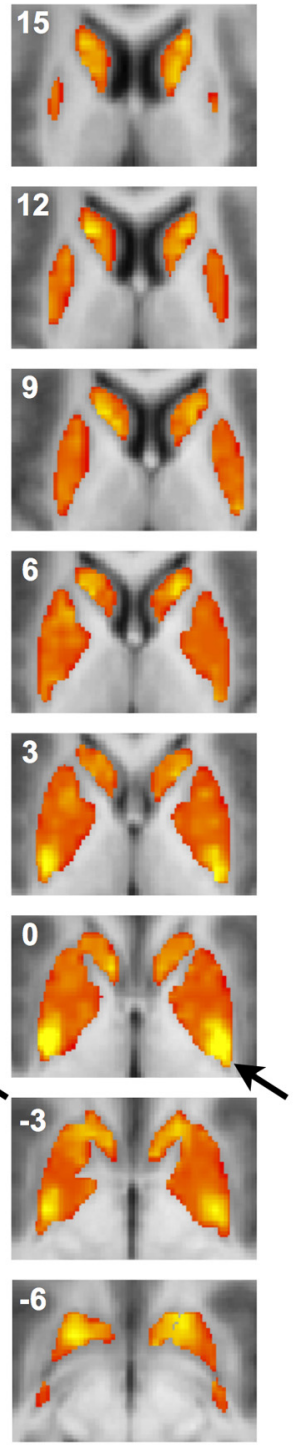

$50 \%$
B adults
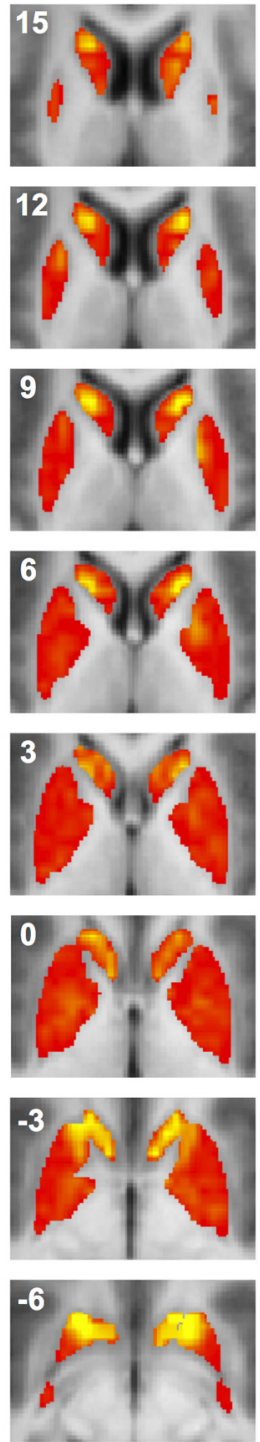

0

Figure 6. A, Reliability of winner assignments in adults and children. Arrows highlight the regions with high reliability in children, but not adults. $\boldsymbol{B}, \Delta r$ value between the "winning" system and the system with the second highest correlation in adults and children. Slices shown in the transverse plane (image left is anatomical left), $z=-6$ to $z=15$ (top left corner).

identified 86 consensus features from the child versus adult cohort 1 classifier (classifier 1) and 85 consensus features from the child versus adult cohort 2 classifier (classifier 2). Each feature represents the partial correlation between a single basal ganglia voxel and a single cortical system, so we calculated the percentage of voxels per cortical system represented by the consensus features. As shown in Figure $7 A$, the systems that contained $>5 \%$ of the voxels in both classifiers included the somatomotor face, auditory, and orbitofrontal systems. In classifier 1 alone, the visual system contained $>5 \%$ of the voxels, and in classifier 2 alone the somatomotor hand and cingulo-opercular systems contained $>5 \%$ of the voxels. However, we will focus on those systems that were similarly represented by both classifiers. Most consensus feature voxels were in the somatomotor face system. Mapping these features onto the brain atlas revealed that the voxels were in the same posterior putamen/pallidum regions identified by the univariate comparison. The auditory system voxels were situated children
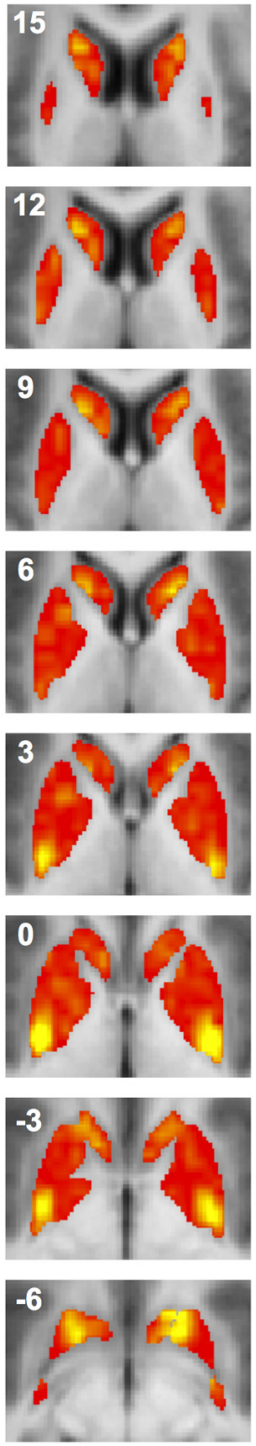

.15 in the dorsal portion of the posterior putamen and pallidum, and were mostly in the left hemisphere. The orbitofrontal system voxels were located bilaterally in the pallidum and ventral striatum.

Since most consensus features overlapped with the univariate results, we ran additional SVMs excluding the voxels that were significant in the univariate analyses, similar to Haxby et al. (2001). Classification accuracy (adult vs child subjects) was $78.3 \%\left(p=5.6 \times 10^{-10}\right.$; sensitivity, $76.7 \%$; specificity, $80 \%)$ for classifier 1 and $75.8 \%\left(p=6.1 \times 10^{-9}\right.$; sensitivity, 68.3\%; specificity, $83.3 \%$ ) for classifier 2 . Interrogation of the features revealed 77 consensus features for classifier 1 and 72 consensus features for classifier 2. Figure $7 B$ shows that the somatomotor hand, orbitofrontal, and auditory systems contained $>5 \%$ of the voxels in both classifiers. Thus, BG functional connectivity with these sensorimotor systems reliably distinguished children and adults, even when the posterior putamen/pallidum voxels identified by the univariate analyses were excluded. Together, these results both underscore the convergence of our univariate and multivariate analyses, and highlight the added value of multivariate methods to reveal aspects of the data that may not be readily apparent by traditional univariate methods.

\section{Discussion}

We examined functional connectivity between the BG and functionally defined cortical systems (Power et al., 2011). Comparing BG-cortical system functional connectivity in children and adults using univariate methods revealed a specific developmental change in a posterior putamen/pallidum region, driven by decreasing functional connectivity with the somatomotor face system from childhood to adulthood. Multivariate methods classified individuals reliably as children or adults based on BG-cortical system functional connectivity. These results demonstrate that rs-fcMRI data are useful for observing functional organization within the human BG, and that this functional organization changes across development. Such findings also have potential implications for future investigations of developmental disorders that involve BG dysfunction.

\section{In adulthood, BG functional organization is consistent with previous connectivity findings}

Adult functional organization within the BG was consistent with the sensorimotor, cognitive, and limbic subdivisions previously described in anatomical studies of human (Lehéricy et al., 2004; Leh et al., 2007; Draganski et al., 2008) and nonhuman primates (Alexander et al., 1986; Parent, 1990; Haber, 2003). Somatomotor regions correlated with the posterior putamen; control/attention systems (encompassing prefrontal regions) correlated with 
A

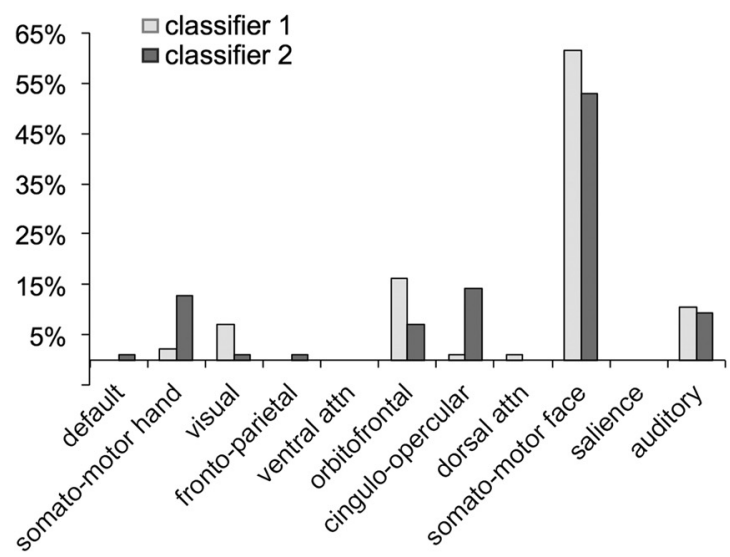

B

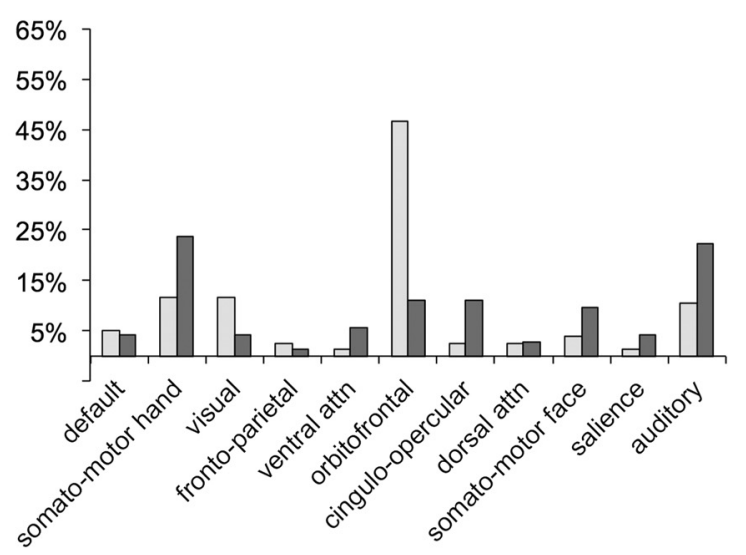

Figure 7. $\quad A, B$, Distribution of consensus features (percentage of all consensus features) by cortical system for classifier 1 (children and adult cohort 1 ) and classifier 2 (children and adult cohort 2) identified by the SVM based on BG - cortical system functional connectivity $(\boldsymbol{A})$ and by the SVM that excluded BG voxels that showed a developmental difference in the univariate analyses ( $\boldsymbol{B}$; i.e., orange voxels in Fig. 3B).

the caudate; and orbitofrontal regions correlated with the ventral striatum. Moreover, the division of somatomotor regions into separate face and hand systems revealed a ventral-to-dorsal topography consistent with anatomical topography in the putamen (Alexander et al., 1986; Flaherty and Graybiel, 1993; Nambu, 2011; Choi et al., 2012).

Our results in adults were also consistent with previous descriptions of functional connectivity in humans. For example, Choi et al. (2012) used a similar methodological approach to describe adult striatal functional organization. As in Choi et al. (2012), we found preferential connectivity in the ventral striatum with the orbitofrontal cortex, in the posterior putamen with somatomotor systems, and in the caudate and anterior putamen with control/attention systems. Minor variations in functional organization between the two studies are likely due to disparities in the predefined cortical systems chosen as a starting point, as we used a graph-theoretical brain system scheme from Power et al. (2011), while Choi et al. (2012) used a clustering algorithmderived partition from Yeo et al. (2011).

Importantly, rs-fcMRI correlations do not necessitate direct anatomical connections (Vincent et al., 2007); thus, functional connectivity can provide novel and distinct information. For example, multiple systems correlated with specific BG nuclei-the orbitofrontal and visual systems correlated with the ventral striatum, and multiple control/attention systems correlated with the caudate nucleus. In addition, the winner-take-all approach identified transitions in functional connectivity that do not correspond to obvious anatomical divisions in BG nuclei. The organization of the salience, ventral attention, and frontoparietal systems in the caudate is not revealed by widely appreciated anatomical boundaries, demonstrating the additional information that a functional approach can provide.

\section{Functional connectivity of the BG shows a developmental change with the somatomotor face cortical system}

Comparisons between children and two distinct cohorts of adults revealed a reliable and notable developmental change: a bilateral cluster of voxels in the posterior putamen and pallidum showed significantly stronger functional connectivity with the somatomotor face system in children than in adults. Functional parcellations identified by the winner-take-all approach demonstrated that voxels in this posterior putamen/pallidum region preferentially correlated with the somatomotor face system in children, but with control/attention systems (ventral attention and frontoparietal) in adults. As correlations with the control/attention systems did not differ significantly in direct statistical comparisons between the age groups, our results suggest that the difference occurs because the functional relationship to somatomotor regions becomes weaker with maturation. Thus, there was decreasing functional connectivity with the somatomotor face system with development (apparent even from 7 to 12 years of age; Fig. $3 C$ ), which appears to give way to preferential functional connectivity with control systems in adulthood. A prominent perspective in the developmental literature is that there is a developmental shift from stimulus-driven to goal-directed processing in the brain (Casey et al., 2005). Therefore, we expected to find a developmental shift in attention/control systems, but instead found a sensorimotor shift.

We are unaware of any research on corticostriatal connectivity from childhood to adulthood in humans with which we can contextualize our findings. One mouse study investigated striatal responses to cortical stimulation in development, specifically comparing mice at postnatal days 28-32 (approximately equivalent to adolescence) to adult mice (Galiñanes et al., 2009). The authors reported decreased striatal responsiveness to motor and prefrontal cortical stimulation over development. Given our results, the decreased responsiveness reported in mice could be specific to somatomotor systems, but further work is needed to test such a hypothesis. Using a "winner-take-all” functional connectivity approach similar to ours, Fair et al. (2010) demonstrated developmental changes in the human thalamus. Functional connectivity increased over development in some subregions but decreased in others, demonstrating the sensitivity of this method for detecting group changes in both directions. They did not include analysis steps to deal with small head movements that have since been shown to introduce artifacts in rsfcMRI data (Power et al., 2012), and they examined functional connectivity between the thalamus and five anatomically defined cortical swaths, rather than the functionally defined cortical systems we used. Therefore, it would be interesting for future work to examine functional connectivity between the thalamus (as well as other subcortical structures, e.g., amygdala) and functional 
cortical systems over the course of development, while optimally accounting for motion.

\section{BG-cortical system functional connectivity reliably predicts maturity}

We applied MVPA, specifically using SVM classifiers, to determine whether patterns in BG-cortical system functional connectivity could predict maturation status. The SVM classified individuals reliably and with high accuracy, demonstrating that our measurement of BG-cortical system functional connectivity contains patterns of information that distinguish children and adults.

One benefit of SVM is the identification of features that contribute highly to the classification. Aside from functional connectivity in the posterior putamen/pallidum with the somatomotor face system, which was expected to distinguish children and adults based on the univariate results, functional connectivity with the orbitofrontal, auditory, and somatomotor hand systems were identified as the next best predictors of maturation status. These results support the idea that MVPA and univariate results, while often broadly consistent, capitalize on different aspects of the same data, as MVPA can reveal additional information not detected by univariate analyses (Jimura and Poldrack, 2012). Notably, control/attention systems were not useful features for binary classification in any SVM, a surprising finding given the evidence for protracted development of cognitive control and related brain regions (e.g., Sowell et al., 1999). One interpretation is that while attention/control systems themselves do develop into adulthood, their functional relationships with the BG do not. Control system-BG functional connectivity may be well developed by 7 years of age, while sensorimotor system-BG functional connectivity continues to undergo developmental changes. Thus, our results highlight the importance of relationships between the BG and non-control/attention systems in development. The specific functional connections with the orbitofrontal, auditory, and somatomotor hand systems may relate to reward processing, language, and fine motor development; however, future research is needed to characterize the developmental trajectories of these specific functional relationships.

\section{Implications}

A major motivation for the present study was to refine a method for interrogating functional organization within the BG that might be useful for studying developmental disorders that involve BG dysfunction. Our main finding of reduced functional connectivity with the somatomotor face system over development strikes a particular chord when considering certain developmental disorders, such as Tourette syndrome (TS). TS is characterized by motor and vocal tics, and is thought to involve dysfunction in cortico-striatal-thalamo-cortical circuits (Mink, 2001; for review, see Greene et al., 2013). Tics develop in childhood and often improve into late adolescence and early adulthood (Leckman, 2003). Interestingly, the most common tics involve orofacial muscles (e.g., blinking, sniffing, throat clearing). Therefore, the selective shift in functional connectivity with the somatomotor face system (encompassing primary motor and somatosensory areas related to the face) may be particularly important for understanding the developmental course of TS. The developmental trajectory of BG functional organization may be atypical in TS, and the partial correlation coefficient between the posterior putamen/pallidum and somatomotor face system could be used to quantify connectivity differences in TS and control subjects.
Additionally, the ability to make predictions on a singlesubject basis using rs-fcMRI data, specifically BG-cortical system functional connectivity, holds promise for applying such classification methods to disordered populations. For disorders involving BG dysfunction, like TS, this approach may be useful for making diagnostic or treatment predictions about individuals (e.g., who will improve with time or respond to treatment). Future work may benefit from studying the functional organization of the BG in persons with TS and other developmental disorders, and applying SVM to make diagnostic predictions.

\section{References}

Alexander GE, DeLong MR (1985) Microstimulation of the primate neostriatum. II. Somatotopic organization of striatal microexcitable zones and their relation to neuronal response properties. J Neurophysiol 53:14171430. Medline

Alexander GE, DeLong MR, Strick PL (1986) Parallel organization of functionally segregated circuits linking basal ganglia and cortex. Annu Rev Neurosci 9:357-381. CrossRef Medline

Aron AR, Durston S, Eagle DM, Logan GD, Stinear CM, Stuphorn V (2007) Converging evidence for a fronto-basal-ganglia network for inhibitory control of action and cognition. J Neurosci 27:11860-11864. CrossRef Medline

Barnes KA, Cohen AL, Power JD, Nelson SM, Dosenbach YB, Miezin FM, Petersen SE, Schlaggar BL (2010) Identifying basal ganglia divisions in individuals using resting-state functional connectivity MRI. Front Syst Neurosci 4:18. CrossRef Medline

Ben-Hur A, Ong CS, Sonnenburg S, Schölkopf B, Rätsch G (2008) Support vector machines and kernels for computational biology. PLoS Comput Biol 4:e1000173. CrossRef Medline

Black KJ, Snyder AZ, Koller JM, Gado MH, Perlmutter JS (2001) Template images for nonhuman primate neuroimaging: 1 . Baboon. Neuroimage 14:736-743. CrossRef Medline

Black KJ, Koller JM, Snyder AZ, Perlmutter JS (2004) Atlas template images for nonhuman primate neuroimaging: baboon and macaque. Methods Enzymol 385:91-102. CrossRef Medline

Bradshaw JL, Sheppard DM (2000) The neurodevelopmental frontostriatal disorders: evolutionary adaptiveness and anomalous lateralization. Brain Lang 73:297-320. CrossRef Medline

Buckner RL, Krienen FM, Castellanos A, Diaz JC, Yeo BT (2011) The organization of the human cerebellum estimated by intrinsic functional connectivity. J Neurophysiol 106:2322-2345. CrossRef Medline

Casey BJ, Tottenham N, Liston C, Durston S (2005) Imaging the developing brain: what have we learned about cognitive development? Trends Cogn Sci 9:104-110. CrossRef Medline

Choi EY, Yeo BT, Buckner RL (2012) The organization of the human striatum estimated by intrinsic functional connectivity. J Neurophysiol 108: 2242-2263. CrossRef Medline

De Martino F, Valente G, Staeren N, Ashburner J, Goebel R, Formisano E (2008) Combining multivariate voxel selection and support vector machines for mapping and classification of fMRI spatial patterns. Neuroimage 43:44-58. CrossRef Medline

Di Martino A, Scheres A, Margulies DS, Kelly AM, Uddin LQ, Shehzad Z, Biswal B, Walters JR, Castellanos FX, Milham MP (2008) Functional connectivity of human striatum: a resting state FMRI study. Cereb Cortex 18:2735-2747. CrossRef Medline

Dosenbach NU, Nardos B, Cohen AL, Fair DA, Power JD, Church JA, Nelson SM, Wig GS, Vogel AC, Lessov-Schlaggar CN, Barnes KA, Dubis JW, Feczko E, Coalson RS, Pruett JR Jr, Barch DM, Petersen SE, Schlaggar BL (2010) Prediction of individual brain maturity using fMRI. Science 329: 1358-1361. CrossRef Medline

Draganski B, Kherif F, Klöppel S, Cook PA, Alexander DC, Parker GJ, Deichmann R, Ashburner J, Frackowiak RS (2008) Evidence for segregated and integrative connectivity patterns in the human basal ganglia. J Neurosci 28:7143-7152. CrossRef Medline

Fair DA, Bathula D, Mills KL, Dias TG, Blythe MS, Zhang D, Snyder AZ, Raichle ME, Stevens AA, Nigg JT, Nagel BJ (2010) Maturing thalamocortical functional connectivity across development. Front Syst Neurosci 4:10. CrossRef Medline

Flaherty AW, Graybiel AM (1993) Two input systems for body representa- 
tions in the primate striatal matrix: experimental evidence in the squirrel monkey. J Neurosci 13:1120-1137. Medline

Fox MD, Zhang D, Snyder AZ, Raichle ME (2009) The global signal and observed anticorrelated resting state brain networks. J Neurophysiol 101: 3270-3283. CrossRef Medline

Galiñanes GL, Taravini IR, Murer MG (2009) Dopamine-dependent periadolescent maturation of corticostriatal functional connectivity in mouse. J Neurosci 29:2496-2509. CrossRef Medline

Gerardin E, Pochon JB, Poline JB, Tremblay L, Van de Moortele PF, Levy R, Dubois B, Le Bihan D, Lehéricy S (2004) Distinct striatal regions support movement selection, preparation and execution. Neuroreport 15: 2327-2331. CrossRef Medline

Graybiel AM (2008) Habits, rituals, and the evaluative brain. Annu Rev Neurosci 31:359-387. CrossRef Medline

Greene DJ, Black KJ, Schlaggar BL (2013) Neurobiology and functional anatomy of tic disorders. In: Tourette syndrome (Martino D, Leckman JL, eds), pp 238-275. Oxford, UK: Oxford UP.

Haber SN (2003) The primate basal ganglia: parallel and integrative networks. J Chem Neuroanat 26:317-330. CrossRef Medline

Haber SN, Knutson B (2010) The reward circuit: linking primate anatomy and human imaging. Neuropsychopharmacology 35:4-26. CrossRef Medline

Haber SN, McFarland NR (1999) The concept of the ventral striatum in nonhuman primates. Ann N Y Acad Sci 877:33-48. CrossRef Medline

Hastie T, Tibshirani R, Friedman J (2001) The elements of statistical learning: data mining, inference, and prediction. New York: Springer.

Haxby JV, Gobbini MI, Furey ML, Ishai A, Schouten JL, Pietrini P (2001) Distributed and overlapping representations of faces and objects in ventral temporal cortex. Science 293:2425-2430. CrossRef Medline

Jimura K, Poldrack RA (2012) Analyses of regional-average activation and multivoxel pattern information tell complementary stories. Neuropsychologia 50:544-552. CrossRef Medline

Lancaster JL, Glass TG, Lankipalli BR, Downs H, Mayberg H, Fox PT (1995) A modality-independent approach to spatial normalization of tomographic images of the human brain. Hum Brain Mapp 3:209-223. CrossRef

Leckman JF (2003) Phenomenology of tics and natural history of tic disorders. Brain Dev 25 [Suppl 1]:S24-S28. Medline

Leh SE, Ptito A, Chakravarty MM, Strafella AP (2007) Fronto-striatal connections in the human brain: a probabilistic diffusion tractography study. Neurosci Lett 419:113-118. CrossRef Medline

Lehéricy S, Ducros M, Van de Moortele PF, Francois C, Thivard L, Poupon C, Swindale N, Ugurbil K, Kim DS (2004) Diffusion tensor fiber tracking shows distinct corticostriatal circuits in humans. Ann Neurol 55:522-529. CrossRef Medline

McClure SM, Berns GS, Montague PR (2003) Temporal prediction errors in a passive learning task activate human striatum. Neuron 38:339-346. CrossRef Medline

Middleton FA, Strick PL (2000) Basal ganglia output and cognition: evidence from anatomical, behavioral, and clinical studies. Brain Cogn 42: 183-200. CrossRef Medline

Mink JW (2001) Basal ganglia dysfunction in Tourette's syndrome: a new hypothesis. Pediatr Neurol 25:190-198. CrossRef Medline

Nambu A (2011) Somatotopic organization of the primate basal ganglia. Front Neuroanat 5:26. CrossRef Medline
Ojemann JG, Akbudak E, Snyder AZ, McKinstry RC, Raichle ME, Conturo TE (1997) Anatomic localization and quantitative analysis of gradient refocused echo-planar fMRI susceptibility artifacts. Neuroimage 6:156167. CrossRef Medline

Parent A (1990) Extrinsic connections of the basal ganglia. Trends Neurosci 13:254-258. CrossRef Medline

Pereira F, Mitchell T, Botvinick M (2009) Machine learning classifiers and fMRI: a tutorial overview. Neuroimage 45:S199-S209. CrossRef Medline

Power JD, Cohen AL, Nelson SM, Wig GS, Barnes KA, Church JA, Vogel AC, Laumann TO, Miezin FM, Schlaggar BL, Petersen SE (2011) Functional network organization of the human brain. Neuron 72:665-678. CrossRef Medline

Power JD, Barnes KA, Snyder AZ, Schlaggar BL, Petersen SE (2012) Spurious but systematic correlations in functional connectivity MRI networks arise from subject motion. Neuroimage 59:2142-2154. CrossRef Medline

Power JD, Mitra A, Laumann TO, Snyder AZ, Schlaggar BL, Petersen SE (2014) Methods to detect, characterize, and remove motion artifact in resting state fMRI. Neuroimage 84:320-341. CrossRef Medline

Rowland DJ, Garbow JR, Laforest R, Snyder AZ (2005) Registration of [18F]FDG microPET and small-animal MRI. Nucl Med Biol 32:567-572. CrossRef Medline

Schölkopf B, Smola AJ (2002) Learning with kernels: support vector machines, regularization, optimization and beyond. Cambridge, MA: MIT.

Selemon LD, Goldman-Rakic PS (1985) Longitudinal topography and interdigitation of corticostriatal projections in the rhesus monkey. J Neurosci 5:776-794. Medline

Shulman GL, Pope DL, Astafiev SV, McAvoy MP, Snyder AZ, Corbetta M (2010) Right hemisphere dominance during spatial selective attention and target detection occurs outside the dorsal frontoparietal network. J Neurosci 30:3640-3651. CrossRef Medline

Sowell ER, Thompson PM, Holmes CJ, Jernigan TL, Toga AW (1999) In vivo evidence for post-adolescent brain maturation in frontal and striatal regions. Nat Neurosci 2:859-861. CrossRef Medline

Van Dijk KR, Sabuncu MR, Buckner RL (2012) The influence of head motion on intrinsic functional connectivity MRI. Neuroimage 59:431-438. CrossRef Medline

Vincent JL, Patel GH, Fox MD, Snyder AZ, Baker JT, Van Essen DC, Zempel JM, Snyder LH, Corbetta M, Raichle ME (2007) Intrinsic functional architecture in the anaesthetized monkey brain. Nature 447:83-86. CrossRef Medline

Wig GS, Laumann TO, Petersen SE (2013) An approach for parcellating human cortical areas using resting-state correlations. Neuroimage. Advance online publication. Retrieved March 20, 2014. doi:10.1016/j.neuroimage.2013. 07.035. CrossRef Medline

Yeo BT, Krienen FM, Sepulcre J, Sabuncu MR, Lashkari D, Hollinshead M, Roffman JL, Smoller JW, Zöllei L, Polimeni JR, Fischl B, Liu H, Buckner RL (2011) The organization of the human cerebral cortex estimated by intrinsic functional connectivity. J Neurophysiol 106:1125-1165. CrossRef Medline

Zhang D, Snyder AZ, Fox MD, Sansbury MW, Shimony JS, Raichle ME (2008) Intrinsic functional relations between human cerebral cortex and thalamus. J Neurophysiol 100:1740-1748. CrossRef Medline 